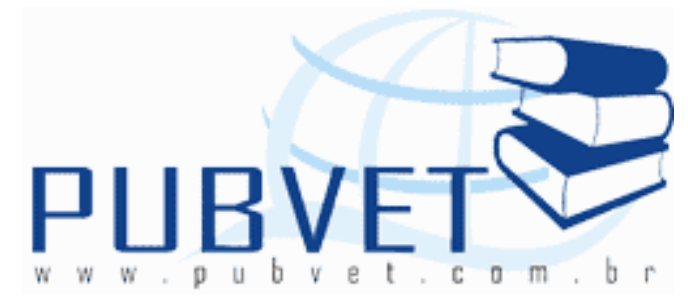

PUBVET, Publicações em Medicina Veterinária e Zootecnia.

\title{
Intervalo de parto e período de serviço em bovinos de leite
}

\section{Gioto Ghiarone Terto e Sousa ${ }^{1}$; Nilton Andrade Magalhães ${ }^{2}$; Leopoldina Almeida Gomes ${ }^{2}$; Hélcio Santos Correia ${ }^{3}$; Severino Cavalcante de Sousa Júnior ${ }^{4}$; Karina Rodrigues Santos ${ }^{4}$; José Elivalto Campelo Guimarães ${ }^{5}$}

${ }^{1}$ Pós-Graduando em Ciência Animal, Universidade Federal do Piauí, UFPI, Teresina.

${ }^{2}$ Docentes, Instituto de Ensino Superior Múltiplo, IESM, Timon.

${ }^{3}$ Médico Veterinário, Especialista em Bovinocultura Leiteira, TECFERTIL, Teresina.

${ }^{4}$ Docentes, Universidade Federal do Piauí, UFPI, Bom Jesus.

${ }^{5}$ Docente, Universidade Federal do Piauí, UFPI, Teresina.

\section{Resumo}

O intervalo entre parto (IEP) é uma das características mais importantes reprodutiva do rebanho leiteiro sendo constituído pelo período seco e de lactação. Diversos de meio ambiente o influência de forma marcante como clima, manejo, controle sanitário. O período seco compreende o período de espera voluntaria e período de serviço (PS). O IEP determina uma maior produção de leite durante a vida útil da vaca enquanto intervalos longos atuam em sentido contrario. Bovino leiteiro manejado em condições adequadas de manejo alimentar e sanitário a duração deste intervalo é de 12 a 14 meses e o PS não deve ultrapassar 90 dias. O estudo teve como objetivo avaliar IEP e PS 
SOUSA, G.G.T. et al. Intervalo de parto e período de serviço em bovinos de leite. PUBVET, Londrina, V. 6, N. 22, Ed. 209, Art. 1398, 2012.

numa fazenda localizada no município de Campo Maior-PI. A pesquisa é um estudo retrospectivo realizada no período de agosto a setembro de 2010 através de dados catalogados em programa computadorizado RAISER VET de controle zootécnico dos anos de 1998 a 2009. Os resultados obtidos foram avaliados e registrados todas as datas de parições e em seguida calculados 0 IEP e PS. Os dados foram tabulados em programa computadorizado em planilha do Excel e analisados estatisticamente através de media aritmética e desvio padrão. A média do IEP foi de 422,05 dias com desvio padrão de 117,51 dias estando esse índice de acordo com o citado na literatura que de 12 a 14 meses. A média do PS 141,98 dias com desvio padrão de 113,31 sendo superior ao citado na literatura que afirma que esse período não deve ultrapassar 90 dias, mas, mesmo assim não ouve influencia negativa do IEP. O elevado OS pode estar associado ao manejo reprodutivo inadequado da fazenda. A fazenda apresenta IEP esperado e PS muito elevado; o manejo inadequado e o PS elevado não refletem negativamente no IEP; a mestiçagem do rebanho parece ser um dos responsáveis pelo elevado PS.

Palavras-chave: bovino leiteiro, intervalo entre parto, período de serviço, rebanho bovino.

\title{
Interval between childbirths and period of service in a Milk property in the city of Campo Maior-PI
}

\begin{abstract}
The interval between childbirth (IEP) is one of the most important features of the dairy herd being constituted reproductive by dry period and lactation. Several of the environment influence so remarkable as climate, management, health control. The dry period comprises the period of expected service period and voluntary (PS). The IEP provides greater milk production during the lifetime of the cow while long intervals work in scented contrary. Dairy cattle held in proper conditions of health and food handling the duration of this range is from 12 to 14 months and the PS should not exceed 90 days. The study
\end{abstract}


SOUSA, G.G.T. et al. Intervalo de parto e período de serviço em bovinos de leite. PUBVET, Londrina, V. 6, N. 22, Ed. 209, Art. 1398, 2012.

aimed to evaluate IEP and PS on a farm located in the municipality of Campo Maior-PI. The search is a retrospective study conducted during the period from August to September 2010 through data catalogued in computerized program RAISER VET zootechnical year's control of 1998 to 2009. The results obtained were assessed and registered all dates of parições and then calculated the IEP and PS. data was tabulated on computerized program in Excel spreadsheet and analysed statistically through media arithmetic and standard deviation. The average of the IEP was 422.05 days with standard deviation of day with this index 117.51 according to the cited in the literature that the 12 months 14 . The average day with standard deviation 141.98 PS of 113.31 than being cited in the literature that States that this period should not exceed 90 days, but you still don't hear the negative influences of the IEP. The high may be linked to the reproductive management of inappropriate farm. The farm presents IEP expected and PS too high; inappropriate handling and the PS high do not reflect negatively on the IEP; the mestizaje of the flock appears to be a responsible high PS.

Keywords: bovine flock, interval between childbirth, milk bovine, period of service.

\section{Introdução}

O intervalo entre partos (IEP) é uma das características mais importantes para a avaliação da eficiência reprodutiva dos rebanhos leiteiros, sendo constituído pelo período de gestação e pelos dias abertos, ou período seco e período de lactação. Os dias abertos compreendem o período de espera voluntária e o período de serviço (VOLACO, 2006). Entretanto, diversos fatores de meio ambiente como variações de clima, manejo, alimentação e controle sanitário o influenciam de forma marcante, os quais têm sido amplamente estudados, tanto em condições de clima temperado quanto em clima tropical.

Segundo Oliveira et al. (1997) e Freitas et al. (1998) o intervalo entre partos é influenciado por fatores de ordem reprodutiva, nutricionais e de 
SOUSA, G.G.T. et al. Intervalo de parto e período de serviço em bovinos de leite. PUBVET, Londrina, V. 6, N. 22, Ed. 209, Art. 1398, 2012.

manejo. Tem-se observado que intervalos curtos entre partos conduzem uma maior produção de leite durante a vida útil da vaca, enquanto intervalos longos atuam em sentido contrário, traduzidos na falta de adaptação dos animais ao meio ambiente. Em bovinos de aptidão leiteira, manejados em condições adequadas de alimentação e cuidados sanitários, a duração ótima deste intervalo está provavelmente entre 12 e 14 meses.

De acordo com Ahmadzadeh (2004), vacas com intervalos entre partos de 12 meses necessitam de mais inseminações para ficarem prenhas do que vacas com intervalos maiores (15 ou 18 meses), pois vacas com intervalos entre partos mais extensos conseguem recuperar a função ovariana cíclica mais facilmente. O desempenho reprodutivo do rebanho e a eficiência têm grande impacto na produção leiteira por lactação, média produtiva durante o tempo de vida da vaca, número de bezerros nascidos e também sobre o lucro da fazenda. O número alto de vacas secas faz com que o custo caia sobre as vacas em lactação, afetando a economicidade do sistema.

O estudo teve como objetivo avaliar o período de serviço (PS) e intervalo de parto (IDP) numa media propriedade leiteira com produção diária de 200 kg/leite/dia, localizada no município de Campo Maior-PI.

\section{Material e Métodos}

A pesquisa é um estudo retrospectivo, sendo realizada no período de agosto a setembro de 2010, através do levantamento de dados que foram catalogados durante os anos de1998 a 2009 através do controle zootécnico utilizado na fazenda, que fica localizada no município de Campo Maior-PI, às margens da BR 343, Km 278, mesorregião do Estado, com altitudes de 125

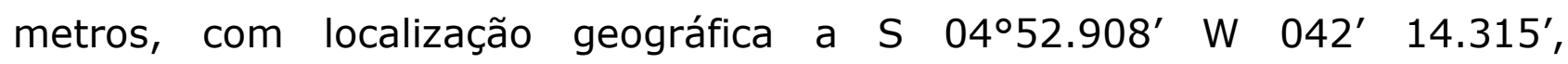
apresentando área total de 500 há.

Foram utilizados dados registrados no programa computadorizado específico de controle zootécnico, RAISER VET. Os resultados obtidos através 
do controle zootécnico adotado na propriedade foram avaliados e registrados todas as datas de parição para serem efetuados os cálculos do intervalo de parto (IDP) e, juntamente com dias de gestação para os cálculos de período de serviço (PS) visando padronizar o conhecimento dos principais índices utilizados na avaliação do desempenho reprodutivo em gado de leite. Para realização dos cálculos dos respectivos índices foram utilizadas fórmulas matemáticas, representadas abaixo, descritas por Ferreira, (1991). Os intervalos de partos (IEP) e período de serviço (PS) foram tabulados em programa computadorizada utilizando-se planilhas do Excel, sendo analisados estatisticamente através de média aritmética e desvio padrão dos índices.

Formula 1: calculo para intervalo Formula 2: calculo para período de entre partos: serviço:

$B 1=A 1 \times 365+M 1 \times 30+D 1$ PS = IEP - 285 (tempo de gestação,

$B 2=A 2 \times 365+M 2 \times 30+D 2$ dias)

$\mathrm{IEP}=\mathrm{B} 2-\mathrm{B} 1$ PS $=80$ dias "ideal"

IEP $=365$ dias "ideal"

$A_{1}=$ Ano do parto $1 ; A_{2}=$ Ano do parto $2 ; M_{1=}$ Mês do parto $1 ; M_{2=}$ Mês do parto $2 ; D_{1}=$ Dia do parto $1 ; D_{2}=$ Dia do parto $2 ; \mathrm{IEP}=$ Intervalo entre parto.

\section{Resultados e Discussão}

A fazenda apresenta uma mestiçagem de raças sendo encontradas girolanda $1 / 2$ sangue, pardo suíço e mestiços de guzerá. As vacas girolanda são utilizadas no programa de inseminação artificial e as outras raças reproduzemse por monta natural.

Verificou-se que a média do intervalo de parto (IDP) da fazenda, no período pesquisado, foi de 422, 05 dias ou 14 meses aproximadamente com desvio padrão de 117, 51 dias. Este índice está de acordo com o que relata a 
SOUSA, G.G.T. et al. Intervalo de parto e período de serviço em bovinos de leite. PUBVET, Londrina, V. 6, N. 22, Ed. 209, Art. 1398, 2012.

literatura segundo afirmam Oliveira et al. (1997) e Freitas et al. (1998) que gado leiteiro apresenta o intervalo de parto (IDP) de 12 a 14 meses.

Com relação ao período de serviço (PS) a média foi 141,98 dias com desvio padrão de 113,31 dias (figura 1). O PS não deve ultrapassar 90 dias a fim de se obter um intervalo de parto (IDP) próximo de 12 meses (Hafez, 2007). Na fazenda pesquisada verificaram-se PS muito alto embora não tenha refletido diretamente no intervalo de parto (IDP) de forma negativa. O elevado período de serviço (PS) verificado pode ser justificado por manejo reprodutivo inadequado, haja vista que na fazenda utiliza-se tanto monta natural quanto inseminação artificial de forma descontrolada.

A relação entre intervalo de parto (IDP) e período de serviço (PS) indica a situação reprodutiva da fazenda, apontando virtudes ou mostrando a gravidade do problema (Hafez, 2007). Através dos resultados obtidos verificou-se uma situação de total descontrole no manejo reprodutivo e o resultado é um período de serviço (PS) elevado dentro da fazenda.

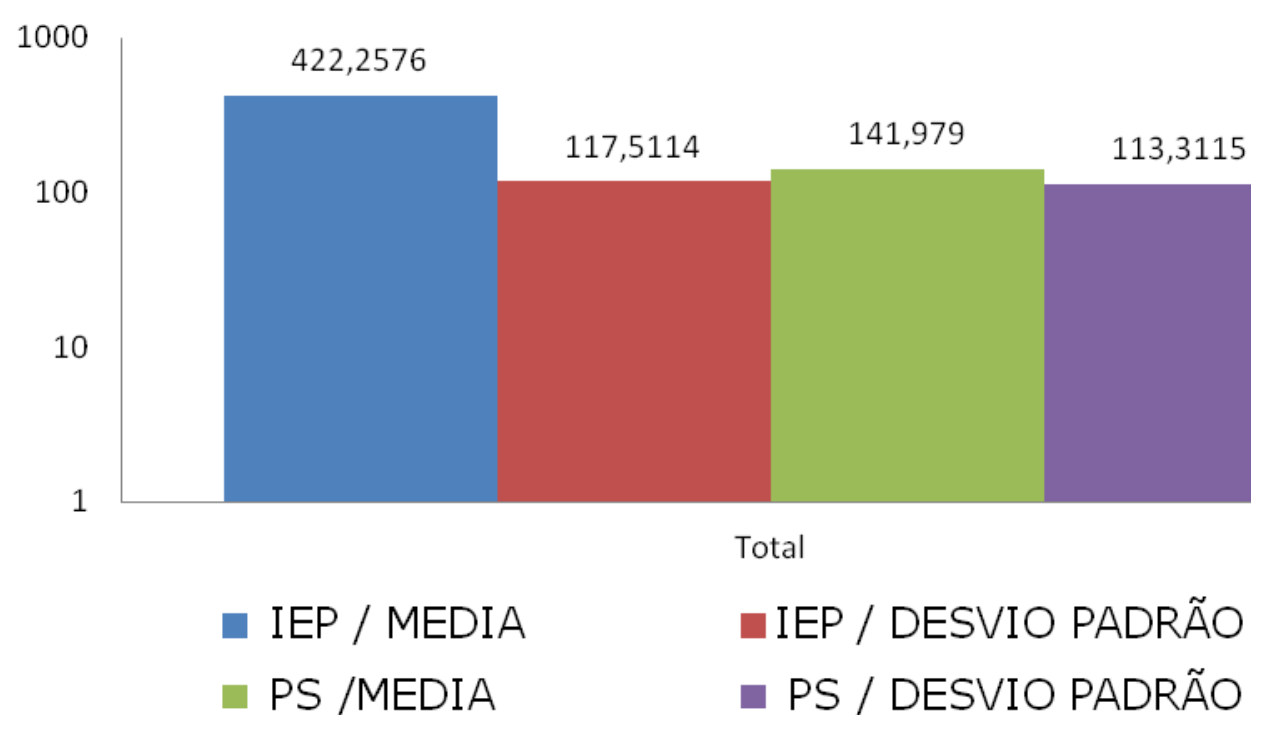

Figura 1. Total de intervalo entre partos e período de serviço, media e desvio padrão dos IEP e PS da fazenda localizada no município de Campo Maior-PI. 
SOUSA, G.G.T. et al. Intervalo de parto e período de serviço em bovinos de leite. PUBVET, Londrina, V. 6, N. 22, Ed. 209, Art. 1398, 2012.

Este resultado mostra que a fazenda apesar de manter um sistema de criação extensivo e por esta numa região de clima tropical consegue manter suas média de intervalo de parto (IDP) dentro do esperado para os índices de bovinos leiteiros na região estudada que é de 14 meses ou 425 dias.

\section{Conclusões}

A fazenda apresenta um intervalo de parto dentro do esperado e período de serviço muito acima do preconizado, onde o ideal é $84 \%$.

O manejo reprodutivo da fazenda inadequado e o período de serviço elevado não refletem de forma negativa no intervalo de parto, pois o mesmo se encontra dentro das medias da região de criação.

O grau de sangue do rebanho parece ser um dos responsáveis do elevado período de serviço da fazenda.

\section{Literatura citada}

AHMADZADEH. A. Reproductive and Efficiency. 2004 Disponível em: <http://www.cowconfortzone.com/Idaho Amin.pdf. > Acesso em 09/08/2008

VOLACO, M. S. Fatores de meio ambiente sobre o intervalo entre partos em rebanhos leiteiros no Paraná. Tese de mestrado, UFPR, Curitiba. Imprensa universitária. 2005. 54p.

OliVeirA, A. I. G.; PEREIRA, I. G.; NEIVA, R. S.; GONÇALVES, T. M.; TEIXEIRA, N. M.; ElER, J. P. Fatores de variação dos períodos de serviço e seco em bovinos da raça holandesa no estado de minas gerais. In: 34. Reunião Anual da Soc. Bras. de Zootecnia, 1997, Juiz De Fora. Anais... Juiz De Fora: SBZ, 1997. v. 1. p. 25-27.

FREITAS, M. A. R; NOGUEIRA, J. R.; GROSSI, S. F. Eficiência de produção e fertilidade de bovinos Girolando monitorados por sistema de informação. In: Reunião da Sociedade Brasileira de Zootecnia, 1998, Botucatu. Anais..., da SBZ, 1998.

HAFEZ, E. S. E. Reprodução animal. 7. ed. São Paulo: Manole, 2004. 